\title{
Association of congenital anomalies of the kidney and urinary tract with those of other organ systems: Clinical implications
}

\author{
Amin J Barakat* \\ Department of Pediatrics, Georgetown University Medical Center, Washington, DC, USA
}

\begin{abstract}
Congenital anomalies of the kidney and urinary tract (CAKUT) occur in 5\%-10\% of the population. About 50\%-60\% of affected patients have malformations of other organ systems including the heart and cardiovascular system, gastrointestinal tract, central nervous system, skeletal system, lung, face, genito-reproductive system, abdominal wall, chromosomal abnormalities, multiple congenital anomalies (MCA) and others. CAKUT is a major cause of chronic kidney disease (CKD) especially in children accounting for about $50 \%$ of cases. CAKUT should be suspected in children with anomalies of other organ systems, MCA, chromosomal aberrations, and in newborns with major abnormalities of the ear lobe. Awareness of this association is essential in the early diagnosis and management of CAKUT to prevent renal damage and chronic kidney disease.
\end{abstract}

\begin{abstract}
Abbreviations: ASD: Atrial septal defect; CAKUT: Congenital anomalies of the kidney and urinary tract; CHD: Congenital heart disease; CKD: Chronic kidney disease; CNS: Central nervous system; CV: Cardiovascular; GI: Gastrointestinal; MCA: Multiple congenital anomalies; PDA: Patent ductus arteriosus; PUV: Posterior urethral valves; UPJ: Ureteropelvic junction; VSD: Ventricular septal defect; VUR: Vesicoureteral reflux.
\end{abstract}

\section{Introduction}

Congenital anomalies of the kidney and urinary tract (CAKUT) are a major cause of morbidity in children. The most common phenotypic forms of CAKUT include renal agenesis or hypoplasia, multicystic dysplastic kidney, hydronephrosis, ureteropelvic junction (UPJ) obstruction, megaureter, duplex ureter, vesicoureteral reflux (VUR), and posterior urethral valves (PUV). CAKUT accounts for $27 \%$ of birth defects [1] and $25 \%$ of prenatally detected malformations. The reported incidence of these anomalies varies depending on the methodology, study design and population. The incidence in the newborn population is $0.48 \%$ to $0.87 \%[1,2]$. Okoronkwo, et al. [3] reported CAKUT in $20 \%$ of children under age 18; PUV accounting for $36 \%$ of the anomalies. Barakat, et al. [4] studied 13,775 consecutive autopsies and found an overall occurrence of CAKUT in 4.6 percent, and $9.5 \%$ in males under age 18 . The anomalies involved the kidneys in $45.1 \%$, ureters $29.1 \%$, renal vessels $12.4 \%$, urethra $5.3 \%$, bladder $4.8 \%$ and fistulas $3.3 \%$. CAKUT arises from disruption of normal nephrogenesis as early as five weeks gestation. The etiology is probably multifactorial and includes chromosomal abnormalities, Mendelian and familial inheritance, known syndromes, teratogenic exposure, and probably an interaction between genetic and environmental factors [2]. Although the majority of anomalies are sporadic, the presence of syndromic phenotypes and familial clustering suggest major genetic abnormalities in renal developmental genes that direct this process $[5,6]$. Understanding the molecular control mechanisms of kidney development has led to a paradigm shift away from classic anatomic theories to the current cell biological and genetic approaches to the etiology of CAKUT [7]. Verbitsky, et al. [8] performed genome-wide analysis of copy number variants $(\mathrm{CNVs})$ and demonstrated that different categories of CAKUT are associated with different underlying CNVs. The identification and further characterization of the genetic drivers in these CNVs are important in understanding the complex etiology of CAKUT, including disease penetrance and phenotypic variability [5]. CAKUT can occur as isolated malformations or in association with anomalies of other organ systems. According to Barakat, et al. [9] 47\% of overall autopsies with CAKUT and $60 \%$ of children under age 18 have malformations of other organ systems. Associated malformations were those of the heart and cardiovascular (CV) system in $25 \%$; gastrointestinal (GI) tract, 18\%; central nervous system (CNS), 10\%; skeletal system, 9\%; lung and face, $7 \%$ each; genito-reproductive system, abdominal wall, and chromosomal abnormalities $4 \%$ each; and others, $12 \%$. Rubinstein, et al. [10] found this association in $10 \%$ of all autopsies and $73 \%$ of autopsies of children under 12 . In newborns with CAKUT reported by Stohl, et al. [2], 34\% had associated malformations of other organ systems, $7 \%$ had chromosomal abnormalities and $16 \%$ had non-syndromic, non-chromosomal multiple congenital anomalies (MCA). The most common non-urinary anomaly in their study was musculoskeletal followed by GI, CV, and CNS. CAKUT is responsible for over $50 \%$ of chronic kidney disease (CKD) in children [11], hence early diagnosis is crucial in the prevention and early treatment. The presence of anomalies of other organ systems

${ }^{\star}$ Correspondence to: Amin J Barakat, Department of Pediatrics, Georgetown University Medical Center, Washington, DC, USA, Tel: 7035324446; Fax: 9142228761; E-mail: aybarakat@aol.com

Key words: CAKUT, chronic kidney disease, chromosomal abnormalities, multiple congenital anomalies

Received: June 26, 2020; Accepted: July 14, 2020; Published: July 27, 2020 
should alert the physician to look for CAKUT. The purpose of this paper is to discuss the clinical implications of the association of CAKUT with anomalies of other organ systems.

\section{Congenital anomalies of the heart and cardiovascular (CV) system}

The urinary and CV systems share a common embryologic origin, the mesoderm, hence an insult to the mesoderm during embryogenesis may cause defects in both organs. Patients with CAKUT may have associated CV anomalies in $25 \%$ to $34 \%$ of cases [9,12]. Adhisivam, et al. [12] reported that CV malformations are tenfold higher in children with CAKUT than in controls. In their series $60 \%$ of the $\mathrm{CV}$ anomalies were ventricular septal defect (VSD) followed by atrial septal defect (ASD) and pulmonary stenosis in 25\% each. In the Barakat, et al. autopsy study [9], patients with CAKUT had associated heart and CV defects in $25 \%$ (patent ductus arteriosus-PDA in $12 \%$, VSD in $8 \%$, valvular defects, defects of large vessels and ASD in 6\% each, patent foramen ovale and coarctation of the aorta in 3\% each and other defects in 6\%).

Agustin, et al. [13] found that $30 \%$ of patients with congenital heart disease (CHD) had CAKUT including renal cysts, renal agenesis, hydronephrosis, VUR, duplicated collecting systems, ectopic and horseshoe kidneys. Both, mouse and human studies provided compelling evidence of a genetic link between CHD and CAKUT. According to the authors, exploring the multiple gene mutations that have been described by the authors in patients with both these defects may yield novel insights into kidney anomalies and the mechanism of both cardiac and renal structural defects. The authors also characterized the non-cardiac anomalies in the CHD mutants and found an unexpected high prevalence of renal abnormalities.

Jiang, et al. [14] found that $7.4 \%$ of children with CHD had concurrent CAKUT. The most frequent anomalies in their patients were hydronephrosis (39.4\%), VUR (25\%), and duplicated collecting duct systems (17.3\%). In this series, the most frequent CHD were ASD (35\%), VSD (32.8\%), and PDA (6.2\%). Their data also confirmed that CAKUT can occur in patients with CHD with a frequency higher than expected in the normal population. Buendia Hernández, et al. [15] studied 434 patients undergoing angiocardiography for the diagnosis of their CHD. $14 \%$ of these patients had CAKUT. All these studies agree with an epidemiological study in the Atlanta metro area, which showed $23 \%$ of $\sim 8,000$ subjects with CHD also had renal abnormalities [16]. There was no correlation between the type of CAKUT and the type of cardiac lesion $[13,14]$. Patients with CAKUT associated with CHD were usually urologically asymptomatic, hence patients with CHD should have routine evaluation of the kidney and urinary tract to identify the presence of CAKUT and start early intervention to reduce the occurrence of CKD and improve outcome [14,15]. Similarly, a thorough cardiac examination should be performed in patients with CAKUT.

\section{Congenital anomalies of the GI tract}

GI anomalies (imperforate anus, Meckel's diverticulum, tracheoesophageal fistula and anomalies of the stomach, intestines, liver, spleen and pancreas occur in $18 \%$ of patients with CAKUT [9]. Stoll, et al. [17] found that $81 \%$ of patients with anorectal malformations have CAKUT. Perlman, et al. [18] reported that anal atresia is 30 -fold more in patients with CAKUT than the general population $(1.2 \%$ vs $0.04 \%)$. The absence of anal mucosa in the posterior perineal triangle in their study was found to be an important sonographic marker for the prenatal diagnosis of anal atresia.
In a prospective study of 106 consecutive patients, with Hirschsprung disease (a rare congenital developmental disorder of the bowel characterized by the absence of nerve ganglia in the wall of the bowel), Pini Prato, et al. [19] found 20.7\% to have associated CAKUT, especially renal hypoplasia, VUR and hydronephrosis. Male to female ratio was 4.5:1. Hofmann, et al. [20] in an extensive review of the literature reported this association in $14.3 \%$. It is reasonable therefore to include renal ultrasound in the routine investigation of patients with Hirschsprung disease and anorectal malformations.

\section{Congenital anomalies of the CNS}

Ten percent of patients with CAKUT may have associated CNS anomalies (brain and spinal cord anomalies, meningomyelocele, spina bifida and hydrocephalus) [9]. Bilateral renal dysplasia may be associated with anencephaly, hydronephrosis, spina bifida, and encephalocele [21]. Lu, et al. [22] described five patients with CNS malformations (thin, hypoplastic, or absent corpus callosum, hydrocephalus or ventriculomegaly, Chiari type I malformation, and tethered spinal cord), associated with ureteral and renal defects (VUR, UPJ abnormalities, bifid and megaureter). The mouse Nfia mutant phenotype and the common features among the reported five human cases indicate that NFIA haploinsufficiency contributes to a novel human CNS malformation syndrome that can also include ureteral and renal defects. Barros, et al. [23] also reported that $30.4 \%$ of fetal CNS defects mainly encephalocele are associated with CAKUT.

\section{Congenital anomalies of the skeletal system}

Congenital skeletal anomalies occur in $9 \%$ of patients with CAKUT including upper extremities and hands (4\%), vertebral column and ribs (4\%), lower extremities and feet (3\%) and hips and pelvis (2\%) [9]. Rai, et al. [24] found $26.7 \%$ of congenital vertebral malformations to have associated CAKUT mostly unilateral renal agenesis, occurring in $11.9 \%$ of patients. Anomalies of the musculoskeletal, CNS, GI, CV and respiratory systems may also occur. There was a statistically significant association between genitourinary and costal abnormalities. The authors suggest that the diagnosis of congenital vertebral anomalies should alert the physician to the possible presence of CAKUT.

The co-occurrence of renal and limb anomalies has been referred to as acrorenal syndrome [25]. Kroes, et al. [26] suggested that the association between limb deformities and renal anomalies is possibly explained by the concept of the acrorenal polytopic developmental field defect. Their data did not yield evidence for the existence of one distinct "syndrome" and concluded that the term "acrorenal syndrome" should be avoided.

\section{Congenital anomalies of the lung and diaphragm}

Patients with abnormalities of the ureters, bladder and renal arteries as well as renal dysplasia and agenesis may also have congenital anomalies of the lungs (hypoplastic lungs and abnormal lung lobes) in $6 \%$ of cases, and those of the diaphragm in 2\% [9]. Pulmonary hypoplasia may result from thoracic compression due to reduced amniotic fluid volume that occurs with severe renal anomalies [27]. In addition, fetal breathing movements which are essential for normal antenatal lung growth, are significantly lower in patients with oligohydramnios. Other factors such as reduction in renal proline production by the kidney may also be responsible for abnormal lung growth. Extralobular sequestration of the lung has also been described in association with renal aplasia. The association of pulmonary hypoplasia and bilateral renal agenesis are characteristic of the Potter's syndrome. 
Congenital diaphragmatic hernia especially when bilateral, may be associated with CAKUT (aplastic, polycystic, horseshoe, double, and ectopic kidney, hydronephrosis, and ectopic intrathoracic kidney).

\section{Congenital anomalies of the face and ears}

Congenital facial anomalies and cleft palate with or without cleft lip occur in $7 \%$ of patients with CAKUT [9]. Both, urogenital and auditory systems develop around the $5^{\text {th }}-8^{\text {th }}$ week of gestation. Thus, an insult to the fetus at this time may result in an anomaly of both organs. Huang, et al. [28] reported auditory abnormalities in 5.3\% of children with unilateral renal agenesis. According to the authors, the severity of the auditory system defects often parallels those of the urogenital system.

Preauricular ear tags (small, skin-colored nodules that can be found anywhere along a line drawn between the tragus to the angle of the mouth) occur in 1.7 per 1000 newborns, and pits (small openings at the anterior margin of the crus of the helix) in 1-5\% [29]. Deshpande and Watson [30] studied 13,136 consecutive newborns and found minor external ear anomalies (preauricular skin tags, preauricular sinuses, ear pits, and misshapen pinnae) in 7.3/1000. Because of the association of ear and renal anomalies, it was recommended in the past to perform a routine renal ultrasound on all patients who have ear pits or tags. Kugelman, et al. [31] reported renal abnormalities among infants who had isolated ear pits or tags in $2.2 \%$ compared to $3.1 \%$ among healthy newborns. The authors as well as Wang, et al. [32] suggested that routine renal ultrasound is not indicated in patients who have isolated ear pits or tags, but should be considered in those who have in addition, three or more minor anomalies, dysmorphic features, genetic syndromes, or family history of deafness, auricular and/or renal malformations. Renal anomalies associated with those of the ears include hydronephrosis, horseshoe kidney, and renal aplasia or hypoplasia.

CAKUT occur in individuals with ear anomalies more than the general population, probably due to the fact that they are often a part of specific multiple congenital anomaly (MCA) syndromes that have high incidence of renal anomalies [32]. A good example is Potter's syndrome which occurs when there is oligohydramnios causing distinct facial features (Potter facies-flattened nose, recessed chin, hypertelorism, low-set abnormal ears), pulmonary hypoplasia and other defects. Renal agenesis is the most common defect associated with Potter's syndrome, but dysplastic polycystic and other renal anomalies may occur.

\section{Multiple congenital anomalies (MCA) syndromes}

CAKUT are associated with over 200 genetic syndromes and should be suspected in patients with MCA [33]. Examples include the CHARGE association which is an autosomal dominant condition encompassing a wide spectrum of anomalies including coloboma of iris or retina, retarded growth and development, heart anomalies, coanal atresia, genital hypoplasia and ear defects. Renal anomalies occur in $25 \%$ of CHARGE patients and include renal ectopia, malrotation, and dysgenesis, horseshoe kidney, hydronephrosis, and VUR [34]. CAKUT also occurs in $27 \%$ of patients with the Townes-Brocks syndrome (an autosomal dominant disorder with multiple malformations including external ear anomalies, hearing loss, preaxial polydactyly, triphalangeal thumbs, and imperforate anus) [35] and 50\% of patients with the Beckwith-Wiedemann syndrome (an autosomal dominant disorder characterized by somatic overgrowth, macroglossia, anterior abdominal wall defects, ear anomalies and macrosomia) [36].

\section{Congenital anomalies of the genito-reproductive system}

The Wolffian and Mullerian ducts develop in anatomical proximity, hence abnormalities of the Müllerian system, ovaries and kidney result from a common embryologic defect. The gonads are formed from the genital ridge and mesonephros and a defect in that area could result in multiple organ defects. Four percent of patients with CAKUT (renal agenesis and dysplasia, horseshoe kidney, and ureter and bladder anomalies) have associated anomalies of the reproductive organs and external genitalia [9]. Heinonen [37] reported $17.3 \%$ of women with Müllerian duct anomalies, mainly major uterine anomalies with concomitant cervical and vaginal anomalies had associated CAKUT particularly unilateral renal agenesis. One out of every three patients with unilateral renal agenesis has a significant anomaly of the uterus, ovaries or vagina [38].

Müllerian anomalies occur in up to $37-60 \%$ of females and $12 \%$ of males with unilateral renal agenesis [39]. Additionally, renal anomalies have been reported in $40 \%$ of patients with Müllerian aplasia and $40 \%$ of women with unicornuate uterus. Females usually have abnormalities of the uterus, vagina, ovaries, fallopian tube, and abnormal external genitalia, while males may have cryptorchidism, seminal vesicle cyst, hypoplastic vas, unilateral prostatic agenesis, cystic testicular dysplasia, and hypospadias. Prenatal detection of unilateral renal agenesis should prompt the physician to look for associated genital abnormalities.

\section{Chromosomal aberrations}

The incidence of CAKUT is much higher in many forms of chromosomal aberrations than in the general population [40]. Chromosomal aberrations occur in about $4-7 \%$ of infants with CAKUT $[9,17]$. Autosomal trisomies (75\% of trisomy $8,33-70 \%$ of trisomy 18 , $50-60 \%$ of trisomy 13 , and $7 \%$ of trisomy 21 ) and monosomies $(40 \%$ in each of $5 p$ - and $18 \mathrm{q}-$ and $33 \%$ in $4 \mathrm{p}-$ ) have a high incidence of CAKUT. Sixty to $80 \%$ of patients with Turner syndrome have CAKUT with horseshoe kidney being the most commonly reported anomaly. Nicolaides, et al. [41] found chromosomal anomalies in $23 \%$ of fetuses with obstructive uropathy. A specific syndrome is usually identified in $24 \%$ of patients with MCA [42]. CAKUT should be suspected in patients with chromosomal aberrations with or without MCA. Since $27-50 \%$ of these malformations are due to chromosomal aberrations and since CAKUT represents $50 \%$ of fetal malformations, genetic studies should be seriously considered in fetuses with CAKUT associated with other malformations [43].

\section{Other anomalies}

Single umbilical artery which is the most common anomaly of the umbilical cord may be associated with CAKUT (cystic renal dysplasia, PUV, urethral atresia and hypoplastic or absent bladder [44]. Potter's sequence and Meckel's syndrome may also occur in patients with single umbilical artery. In addition to CAKUT, anomalies of the GI, CV, musculoskeletal and CNS and limb reduction defects may also occur.

Supernumerary nipples which occur in $0.22-6 \%$ of the population may be also associated with CAKUT $[45,46]$. While there is discrepancy in the literature regarding the frequency of association of supernumerary nipples and renal anomalies, most authors seem to agree that the random finding of isolated supernumerary nipples probably does not justify renal investigation $[46,47]$. However, investigation seems to be justified when urinary tract infection or MCA are present [33].

\section{Conclusion}

CAKUT can occur as isolated malformations or in association with anomalies of other organ systems including those of the heart and CV system, GI tract, CNS, skeletal system, lung, face, genito-reproductive system, abdominal wall, chromosomal abnormalities, MCA and others. CAKUT should be suspected in children with anomalies of 
other organ systems, MCA, chromosomal aberrations and in newborns with major abnormalities of the ear lobe. Awareness of this association is essential in the management of affected patients as early intervention contributes to prevention of renal damage and CKD.

\section{References}

1. Egbe A, Uppu S, Lee S, Stroustrup A, Ho D, et al. (2015) Congenital malformations in the newborn population: A population study and analysis of the effect of sex and prematurity. Pediatr Neonatol 56: 25-30.

2. Stoll C, Dott B, Alembik Y, Roth MP (2014) Associated non-urinary congenital anomalies among infants with congenital anomalies of the kidney and urinary tract (CAKUT). Eur J Med Genet 57: 322-328.

3. Okoronkwo NC, Mudi A, Levy C, Khumalo T, Moonsamy G (2020) Congenital anomalies of the kidney and the urinary tract in a South African paediatric nephrology setting. S Afr J Child Health 14: 40-44.

4. Barakat AY, Drougas JG (1991) Occurrence of congenital abnormalities of the kidney and urinary tract in 13,775 autopsies. Urology 38: 347-350.

5. Knoers NVAM, Renkema KY (2019) The genomic landscape of CAKUT; you gain some, you lose some. Kid Intern 96: 267-269.

6. Vivante A, Hildebrandt F (2016) Genetics of congenital anomalies of the kidney and urinary tract. In Barakat AJ, Rushton HG (Editors): Congenital Anomalies of the Kidney and Urinary Tract: Clinical Implications in Children. Switzerland: Springer International Publishing, pp 303-322.

7. Ichikawa I, Kuwayama F, Pope JC, Stephens FD, Miyazaki Y (2002) Paradigm shift from classic anatomic theories to contemporary cell biological views of CAKUT. Kidney Int 61: 889-898.

8. Verbitsky M, Westland R (2019) The copy number variation landscape of congenital anomalies of the kidney and urinary tract. Nat Genetics 51: 117-127.

9. Barakat AJ, Drougas JG, Barakat R (1988) Association of congenital abnormalities of the kidney and urinary tract with those of other organ systems in 13,775 autopsies. Child Nephrol Urol 9: 269-272.

10. Rubinstein M, Meyer R, Bernstein J (1961) Congenital abnormalities of the urinary system. I. A postmortem survey of developmental anomalies and acquired congenital lesions in a children's hospital. J Pediatr 58: 356-366.

11. Ardissino G, Dacco V, Testa S, Bonaudo R, Claris-Appiani A, et al. (2003) Epidemiology of chronic renal failure in children: data from the ItalKid project. Pediatrics 111: e382-7.

12. Adhisivam B, Prahlad N, Vijayakumar M, Nammalwar BR, Muralinath S (2005) Cardiovascular malformations associated with urinary tract anomalies. Indian $J$ Nephrol 15: 8-9.

13. Agustin JTS, Granath K, Panigrahy A, Stewart E, Devine W, et al. (2016) Genetic link between renal birth defects and congenital heart disease. Nat Commun 7:11103.

14. Jiang D, Wang Q, Shi Z, Sun J (2020) Congenital Anomalies of the Kidney and Urinary Tract in Children with Congenital Heart Defects. Kidney Blood Press Res 45: 307-313.

15. Buendía Hernández A, Vázquez J, Fuentes J, Attié F, Ovseyevitz J (1987) Anomalies of the urinary tract associated with congenital heart diseases. Arch Inst Cardiol Mex 57: 207-211.

16. Miller A, Riehle-Colarusso T, Alverson C J, Frias J L, Correa A (2011) Congenital heart defects and major structural noncardiac anomalies, Atlanta, Georgia, 1968 to 2005. J Pediatr 159: 70-78.

17. Stoll C, Alembik Y, Dott B, Roth MP (2007) Associated malformations in patients with anorectal anomalies. Eur J Med Genet 50: 281-290.

18. Perlman S, Bilik R, Leibovitch L, Katorza E, Achiron R, et al. (2014) More than a gut feeling -sonographic prenatal diagnosis of imperforate anus in a high-risk population. Prenatal diagnosis 34: 1307-1311.

19. Prato AP, Rossi V, Mosconi M, Holm C, Lantieri F, et al. (2013) A prospective observational study of associated anomalies in Hirschsprung's disease. Orphanet $J$ Rare Dis 8: 184 .

20. Hofmann AD, Duess JW, Puri P (2014) Congenital anomalies of the kidney and urinary tract (CAKUT) associated with Hirschsprung's disease: a systematic review. Pediatr Surg Int 30: 757-761.

21. Rimoin DL, Pyeritz RE, Korf B (editors) (2013) Emery and Rimoin's Principles and Practice of Medical Genetics, Elsevier Ltd. Chapter 62, p. 17.

22. Lu W, Quintero-Rivera F, Fan Y, Alkuraya FS, Donovan DJ, et al. (2007) NFIA haploinsufficiency is associated with a CNS malformation syndrome and urinary tract defects. PLoS Genet 3: e80.
23. Barros ML, Alvarenga Fernandes D, Vieira de Melo E, Santos Porto RL, Andrade Maia MC, et al. (2012) Central nervous system malformations and associated defects diagnosed by obstetric ultrasonography. Radiol Bras 45: 309-314.

24. Rai AS, Taylor TKF, Smith GHH, Cumming RG, Plunkett-Cole M (2002) Congenita abnormalities of the urogenital tract in association with congenital vertebral malformations. J Bone Joint Surg 84: 891-895.

25. Natarajan C, Jeyachandran D, Subramaniyan B, Thanigachalam D, Rajagopalan A (2013) Congenital anomalies of the kidney and hand: a review. Clin Kidney J 6: 144-149.

26. Kroes HY, Olney RS, Rosano A, Liu Y, Castilla EE, et al. (2004) Renal defects and limb deficiencies in 197 infants: is it possible to define the "acrorenal syndrome"? Am J Med Genet 129A: 149-155.

27. May C, Greenough A (2006) Pulmonary hypoplasia and congenital renal anomalies. Arch Med Sci 1: 6-9.

28. Huang H-M, Yeh R-M, Tan C-T, Chao M-C, Lin K-N (2001) Auditory abnormalities associated with unilateral renal agenesis. Int J Pediatr Otorhinolaryngol 60: 113-118.

29. Adam M, Hudgins L (2003) The importance of minor anomalies in the evaluation of the newborn. NeoReviews 4: e99-e104.

30. Deshpande SA, Watson H (2006) Renal ultrasonography not required in babies with isolated minor ear anomalies. Arch Dis Child Fetal Neonatal Ed 91: F29-30.

31. Kugelman A, Tubi A, Bader D, Chemo M, Dabbah H (2002) Preauricular tags and pits in the newborn: the role of renal ultrasonography. J Pediatr 141: 388-391.

32. Wang RY, Earl DL, Ruder RO, Graham Jr JM (2001) Syndromic ear anomalies and renal ultrasounds. Pediatrics 108: E32.

33. Barakat AJ, Rushton HG (Editors): Congenital Anomalies of the Kidney and Urinary Tract: Clinical Implications in Children. Switzerland: Springer International Publishing, 2016.

34. Blake KD, Davenport SLH, Hall BD, Hefner MA, Pagon RA, et al. (1998) CHARGE Association: an update and review for the primary pediatrician. Clin Pediatr 37: 159-173.

35. Powell CM, Michaelis RC (1999) Townes-Brocks syndrome. J Med Genet 36: 89-93.

36. Mussa, A, Peruzzi L, Chiesa N, De Crescenzo A, Russo S, et al. (2012) Nephrological Findings and Genotype-Phenotype Correlation in Beckwith-Wiedemann Syndrome. Pediatr Nephrol 27: 397-406.

37. Heinonen PK (2018) Renal tract malformations associated with Mullerian duct anomalies. Clin Obstet Gynecol Reprod Med 4: 1-5.

38. Barakat AJ (2002) Association of unilateral renal agenesis and genital anomalies. Case Rep Clin Pract Rev 3: 57-60.

39. Thompson DP, Lynn HB (1966) Genital anomalies associated with solitary kidney. Mayo Clinic Proc 41: 538-548.

40. Barakat AY, Butler MG (1987) Renal and urinary tract abnormalities associated with chromosome aberrations. Int J Pediatr Nephrol 8: 215-226.

41. Nicolaides KH, Rodeck CH, Gosden CM (1986) Rapid karyotyping in non-lethal fetal malformations. Lancet 1: 283-287.

42. Polani PE (1977) Delineation of malformation syndromes. In: Littlefield JW, de Grouchy J, editors. Birth defects. Amsterdam: Excerpta Medica, 422-425.

43. Barakat AJ (2016) Association of congenital anomalies of the kidney and the urinary tract with those of other organ systems. In: Barakat AJ, Rushton HG: Congenital anomalies of the kidney and urinary tract: Clinical implications in children Springer pp: 323-335.

44. Heifetz SA (1984) Single umbilical artery: a statistical analysis of 237 autopsy cases and review of literature. Perspect Pediatr Pathol 8: 345-378.

45. Brown J, Schwartz RA (2003) Supernumerary nipples: an overview. Pediatr Derm 71: 344-346.

46. Mimouni $\mathrm{F}$ (1986) Association of supernumerary nipples and renal anomalies. AJDC 142: 591-592.

47. Hoyme HF (1987) Minor malformations: significant or insignificant? AJDC 141: 947.

Copyright: (C)2020 Barakat AJ. This is an open-access article distributed under the terms of the Creative Commons Attribution License, which permits unrestricted use, distribution, and reproduction in any medium, provided the original author and source are credited. 